\title{
TESTING THE CORRELATION BETWEEN OCCUPATIONAL STRESS AND OCCUPATIONAL BURNOUT AMONG NURSES WORKING IN PUBLIC HEALTH INSTITUTIONS
}

\section{AUTHORS}

KORESPONDENT

Oliver Bojčeski

Health Center,

Regional Center for Sports Medicine, Struga, Macedonia

Oliver Bojčeski ${ }^{1}$, Milivoje Galjak ${ }^{2}$, Ljiljana Kulić ${ }^{2}$,

Slađana Đurić ${ }^{2}$, Momčilo Mirković ${ }^{2}$, Jovana Milošević ${ }^{2}$

${ }^{1}$ Health Center, Regional Center for Sports Medicine, Struga, Macedonia

${ }^{2}$ Faculty of Medicine, University of Priština, Kosovska Mitrovica, Serbia

\section{SUMMARY}

INTRODUCTION: Stress at work is a major problem in the modern world in many areas of work, especially in health care. Occupational burnout of nurses represents a serious problem, not only for themselves. It also affects the quality of the health services provided to patients.

OBJECTIVE is to examine the connection between occupational stress and occupational burnout among nurses working in public health institutions.

METHODS: An examination was carried out to determine aconnection between occupational burnout and occupational stress among nurses working in the hospital, at the departments of surgery, gynecology and emergency. For the evaluation of professional burnout among nurses, the Maslach Burnout Inventory, MBI-HSS pattern, was used, and Matteson and Ivancevich Questionnaire was used to assess occupational stress. Statistical data processing was done by the SPSS 17 software.RESULTS A total of 60 examinees were included in the study, 20 of them from the Surgery, Gynecology and Emergency department. Of these, $51(81.7 \%)$ arefemale and $9(18.3 \%)$ are male. The average age of nurses is 41.45 years, from which 40.9on Surgery; 41.8 on Emergency; 41.65 on Gynecology, and the average years of serviceare 17.91. Descriptive statistics for the examined variables are shown and it can be seen that there is a low level of occupational stress, i.e. the result is below 21 .In the occupational burnout variable, respondents show the medium level of emotional exhaustion $(M=24.05)$, low level depersonalization $(M=3.83)$ and low level of lack of professional achievement subscale $(M=38.71)$. The test results confirm the first hypothesis $(\mathrm{H} 1)$ that with the increaseof occupational stress levels, the level of occupational burnout among nurses also increases. Occupational stress correlates with occupational burnout ( $r=0.529$, $\mathrm{p}<0.01)$, i.e. nurses who have higher results of occupational stress, have more occupational burnout results also. The results of the tests involving correlations between occupational burnout and occupational stress dimensions show that with the increase of occupational stress the dimension of emotional exhaustion also increases $(r=0.493, p<0.01)$, that there is no significant correlation between occupational stress and the dimension of depersonalization, and that the dimension of occupational stress and the dimension of individual realization have significant connection $(r=0.393, p=0.01)$. The results show that there is no statistically significant difference between departments when it comes to occupational burnout, i.e. nurses working in Surgery do not have higher results of occupational burnout than nurses working in Gynecology and Emergency $\mathrm{F}(0.679) \mathrm{p}>0.05$, and that there is no statistically significant difference between the departments in terms of occupational stress, i.e. nurses working in Surgery do not have higher occupational stress results than ones working in Gynecology and Emergency department $F(0.722) p>0.05$. The results do not show significant differences in the level of occupational burnout and occupational stress in relation to nurses' age and years of service.

DISCUSSION AND CONCLUSION: The results of the study show that with increased occupationalstressoccupational burnout also increases and that nurses have low levels of occupationalstress, low depresonization, low level of lack of personal achievement and a medium level of emotional exhaustion. There were no significant differences in occupationalburnout and occupationalstress between nurses working in surgery, gynecology and emergency departments. No significant differences in occupationalburnout and occupationalstress were found in relation to the age and years of service of nurses working in the departments of surgery, gynecology and emergency.

KEYWORDS: nurses, occupational stress, occupational burnout, age, years of service. 


\section{INTRODUCTION}

Occupational stress is a serious problem in some professions, and various studies have shown that health workers have an increased degree of depression and anxiety associated with stress at work. The negative consequences of this are many, among which the most relevant are: professional burnout, absenteeism, job abandonment, reduced patient satisfaction, as well as mistakes in diagnosis and treatment. One of the common problems faced by health workers due to long-term stress is occupational burnout or the burnout syndrome. Occupational burnout is a serious problem, because it also affects the quality of health services provided to patients. When presented through a multidimensional model, it consists of three main components: emotional exhaustion, depersonalization and the lack of personal accomplishment. Occupational burnout is common in professions that are characterized by constant contact with people (1). Some authors define occupational burnout as a psychological syndrome of emotional exhaustion, depresonization, and low personal achievements that emerge as an individual's response to emotional interpersonal stress, most often as a reaction to chronic interpersonal stressors in the working environment. Three dimensions of this reaction are: excessive exhaustion, feeling of cynicism, dismissal in work and ineffectiveness with lack of accomplishment. Factors on which occupationalburnout depends are: factors related to work (a working place, work conditions, etc.) and individual factors (demographic characteristics, personality characteristics, attitude towards the work place, etc.) (2).

\section{OBJECTIVE}

The aim of this paper is to examine the connection between occupational stress and occupational burnout among nurses working in public health institutions.

\section{MATERIAL AND METHODS}

RESEARCH VARIABLES - Occupational stress, i.e. the level of stress that the employee feels evenafter the end of the working day is evaluated by theMatteson-Ivanchevich questionnaire. Occupational burnout, i.e.the feeling of emotional exhaustion, depersonalisation and lack of personal achievements as a result of long-lasting dissatisfaction at work with high emotional demands is measured by the $\mathrm{MB}$ (Maslach Burnout Inventory) questionnaire. Emotional exhaustion: indicators indicating the presence of emotional "weariness" in working with patients. Depersonalization: indicators indicating the presence of negativism towards work and cynicism towards patients. Personal achievement: assessment of one's own work and satisfaction with it.

RESEARCH HYPOTHESES - In accordance with previous relevant research, in which the connection between occupational burnout and occupational stress was found, in response to the research problem two research hypotheses are set: $H_{1}$ and $H_{2}$, both with three sub-hypotheses $\left(H_{1.1}, H_{1.2}, H_{1.3}\right.$ and $\mathrm{H}_{2.1}, \mathrm{H}_{2.2}, \mathrm{H}_{23}$ ). The first research hypothesis $\mathrm{H}_{1}$ : As the level of occupational stress increases, the level of occupational burnout among nurses also increases. Sub-hypothesis $\mathrm{H}_{1.1}$ : As the level of occupational stress increases, the level of emotional exhaustion among nurses also increases. Sub-hypothesis $\mathrm{H}_{12}$ : As the level of occupational stress incre- ases, the level of depersonalization among nurses also increases. Sub-hypothesis $\mathrm{H}_{1.3}$ : As the level of occupational stress increases, the level of lack of personal achievement among nurses is reduced. The second research hypothesis $\mathrm{H}_{2}$ : There is a difference in the level of occupational stress and the level of occupational burnout between nurses working in different departments, who are of different ages and have different work experience. Sub-hypothesis $\mathrm{H}_{2.1}$ : Nurses working at the Surgery department have a higher level of occupational burnout and occupational stress than nurses working at the Gynecology and Emergency department. Sub-hypothesis $\mathrm{H}_{22}$ : Older nurses have a lower level of occupational burnout and occupational stress than younger nurses. Subhypothesis $\mathrm{H}_{23}$ : Nurses with more years of service (work experience) have a lower level of occupational burnout and occupational stress than nurses who have less years of service behind them.

MEASURING INSTRUMENTS - A 6-item Matteson \& Ivanchevich questionnaire was used to assess occupational stress among nurses. Respondents respond using the Likert scale, answering each question from 1 to 7 . The higher the result, the higher the level of occupational stress. Persons with a score below 21 have a low occupational stress level, and those with a score above 22 have a high level of professional stress. Persons with high results in this test report that they have health problems, stay away from work, visit a doctor more often, often use tranquilizers and think they are working and earning less than they can because they do not feel good. The variability coefficient in this study: $\alpha=0.80$.

Maslach Burnout Inventory, in particular MBI-HSS (Human Service Survey), was used to assess occupational burnout in nurses, specifically for healthcare and social care professions. Responses are given on a scale that expresses the frequency of the view or relationusing seven degrees, ranging from 0 - Never to 6 - Daily. The MBI contains 22 statements that are arranged in three dimensions. Emotional exhaustion presents emotional weariness in working with clients/patients (9 questions numbered 1, 2, 3, 6, 8, 13, 14, 16 and 20 ) and qualifies as High emotional exhaustion $\geq 27$; Medium emotional exhaustion between 17-26 and Low emotional exhaustion between 0-16. Depersonalization is the appearance of negative attitude and cynicism towards clients/patients (5 questionsnumbered 5, 10, 11, 15 and 22) and qualifies as High Depersonalization $\geq 13$; Medium Depersonalization between 7-12 and Low Depersonalization between $0-6$. The lack of personal accomplishment is a negative assessment of one's own work (8 questionsnumbered $4,7,9,12,17,18,19$ and 21), and qualifies as High deficiency of personal achievement between 0-31; Medium deficiency of personal achievement between 32-38 and Low deficiency of personal achievement $\geq 39$. The result of occupational burnout is obtained by adding the answers received for each question, separately for each scale. Emotional exhaustion and depersonalisation have a reverse interpretation of results as opposed to a personal accomplishment, i.e. in the first two, they are desirable as lower values, and in the third scale, the highest values are more desirable. The variability coefficient in this study: $\alpha=0,766$.

The research involved a total of 60 respondents, nurses employed in JZU "General Hospital" in the city of Struga, Macedonia. The nurses that participated in the research work at the following departments: Surgery (20), Emergency (20) and Gynecology (20). The selection of departments was designed to achieve greater heterogeneity of the specimen and to make a comparison between them. The survey was 
conducted in January 2017. on three occasions, separately in each department. Each respondent was provided with a copy of both questionnaires, which took about 10 minutes to be completed. Although the questionnaires themselves contain a guide, still the respondents were guided how to fill the questionnaires, they were promised anonymity of the results and indicated that its purpose is solely for research intents. The respondents were then informed with the ethical rules, respect of the anonymity of their responses and the importance of their answers being honest. Respondents were stressed that they can ask questions if some of the claims are not clear to them.

Statistical data processing was done by the SPSS 17 software. In the beginning, descriptive statistics for the tested variables (arithmetic mean, standard deviation, minimum and maximum results) were made. Then theresearch hypotheses were tested, i.e. the first research hypothesis $\mathrm{H}_{1}$ and its sub-hypotheses were verified by Pearson correlation coefficient, while the second research hypothesis $\mathrm{H} 2$ and its sub-hypotheses were tested with the corresponding ANOVA equation and the T-test.

\section{RESULTS}

The survey involved a total of 60 respondents (nurse/ technician), i.e. 20 from the Surgery, 20 from the Emergency and 20 from the Gynecology department. Of these, 51 $(81.7 \%)$ female and $9(18.3 \%)$ male. The average age of respondents is 41.45 years, on Surgery 40.9 , Emergency 41.8 and Gynecology 41.65 years old, and the average age of service is 17.91 .

Descriptive statistics ofexamined variables in this study are shown in Table 1. It is clear that there is a low level of occupational stress, i.e. a score below 21. In the occupational burnoutvariable, the respondents show a medium level of emotional exhaustion $(M=24.05)$, low depersonalization $(M=3.83)$ and the low level of the subscale lack of professional achievement $(M=38.71)$. The mesokurtosisdistribution of the results is with a greater representation of the result of about 0 . The calculated skewness indicates the distribution of the results, that it is normal and symmetric, i.e. all variables are normally distributed (Table 1).

Table 1. Descriptive statistics of examined variables

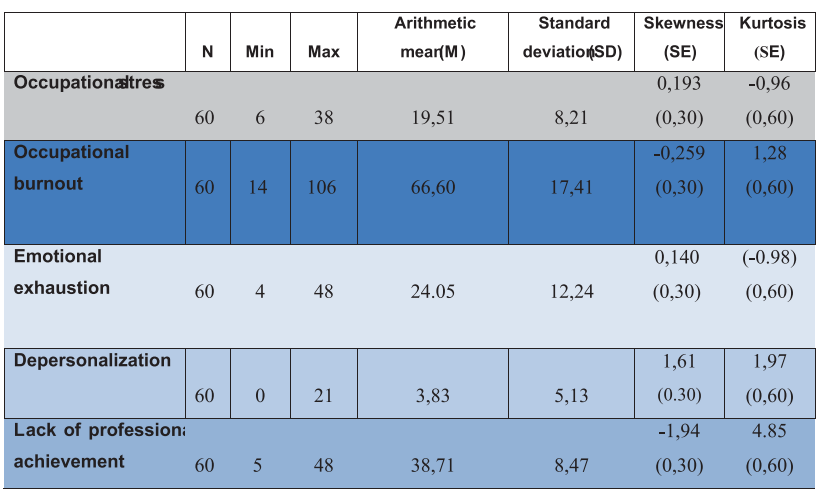

Table 2 presents the results of testing the first research hypothesis $\mathrm{H}_{1}$ : As the occupational stress level increases, the level of occupational burnout among nurses also increases. Findings of the Pearson coefficient application on the overall sample show that occupational stress correlates with occu- pational burnout $(r=0.529, p<0.01)$, that is, nurses that have higher results of occupational stress, have higher results of occupational burnout, which confirms the first research hypothesis. The testing results of the sub-hypotheses $\mathrm{H}_{1.1}, \mathrm{H}_{1.2}$ and $\mathrm{H}_{1.3}$ involving correlations between occupational burnout and occupational stress dimensions show that with the increase of occupational stress, the emotional exhaustion rate increases $(r=0.493, p<0.01)$, while no significant correlationbetween occupational stress and the dimensions of depersonalization was found. Finally, there is significant correlation between the dimension of occupational stress and the lack of professional achievement dimension( $r=0.393$, $p=0.01$ ). Consequently, the sub-hypothesis $H_{1.1}$ ha been confirmed, the sub-hypothesis $\mathrm{H}_{12}$ has not been confirmed and the sub-hypothesis $\mathrm{H}_{1.3}$ has been confirmed.

Table 2. Correlation between occupational burnout and its dimensions withoccupational stress

\section{Occupational stress}

\begin{tabular}{ll}
\hline Occupational burnout & $\mathrm{r}=0.529 * *$ \\
\hline Emotional exhaustion & $\mathrm{r}=0,493^{* *}$ \\
Depersonalization & $\mathrm{r}=0,30$ \\
Lack of professional achievement & $\mathrm{r}=0,393 * *$ \\
\hline$* * \mathrm{p}<0.01$, & \\
\hline
\end{tabular}

Table 3 and Table 4 show the results of testing the first sub-hypothesis $\mathrm{H}_{2.1}$ : Nurses working in Surgery have higher results of occupational burnout and occupational stress than nurses working in Gynecology and Emergency departments. This sub-hypothesis is related to the postulates of the second research hypothesis $\mathrm{H} 2$ for existence of differences in the examined variables by department, age and work experience (years of service). In Table 3, the one-way ANOVA (Analysis of variance) $\mathrm{F}$ does not exceed the critical value of significance, which means that there is no statistically significant difference between departments in regard to occupational burnout, i.e. nurses working in Surgery do not have higher results of occupational burnout than nurses working in Gynecology and Emergency, F(0.679), p> 0.05.

Table 3. ANOVA results for differences in occupational burnout among nurses from different departments

\begin{tabular}{lcc} 
Source & $\mathbf{F}$ & $\mathbf{P}$ \\
Between group: & 0,679 & 0,511 \\
\hline
\end{tabular}

Table 4. ANOVA results for differences in occupational stress among nurses from different departments

\begin{tabular}{lcc} 
Source & $\mathbf{F}$ & $\mathbf{P}$ \\
Between group: & 0,722 & 0,490 \\
\hline
\end{tabular}

In Table 4, the one-way ANOVA F does not exceed the critical value of significance, which means that there is no statistically significant difference between departments in regard to occupational stress, i.e. nurses working in Surgery do not have higher results of occupational stress than nurses working in Gynecology and Emergency, $F(0.722)$, $p>0.05$.

Therefore, sub-hypothesis $\mathrm{H}_{2.1}$ : Nurses working in Surgery have higher results of occupational burnout and 
occupational stress than nurses working in Gynecology and Emergency has not been confirmed / is discarded.

Table 5 gives the results ( $M, S D$ ) for the variables of occupational burnout and occupational stress for nurses of all three departments: Surgery, Gynecology and Emergency. According to arithmetic mean $(M)$, nurses working in Surgery $(\mathrm{N}=20)$ have the highest level of occupational burnout and occupational stress, while nurses working in Emergency $(\mathrm{N}=20)$ have the lowest results.

Table 5. Descriptive statistics of occupational stress and occupational burnout of nurses from different departments

\begin{tabular}{lcccc}
\hline & $\begin{array}{c}\text { Gynecology } \\
\mathbf{N = 2 0}\end{array}$ & $\begin{array}{c}\text { Surgery } \\
\mathbf{N = 2 0}\end{array}$ & $\begin{array}{c}\text { Emergency } \\
\mathbf{N = 2 0}\end{array}$ & $\begin{array}{c}\text { Arithmetic } \\
\text { mean(M) }\end{array}$ \\
& $\mathbf{M}$ (SD) & $\mathbf{M}(\mathbf{S D})$ & $\mathbf{M}(\mathbf{S D})$ & \\
Occupationælurnout & $\mathbf{M ~} 67,40$ & $\mathbf{M} 69,35$ & $\mathbf{M} 63,05$ & 66.60 \\
& $\mathbf{S D}(15,08)$ & $\mathbf{S D}(9,30)$ & $\mathbf{S D}(24,61)$ & \\
Occupationalstress & 18,90 & 21,30 & 18,35 & 19.51 \\
& $(6,46)$ & $(7,07)$ & $(10,61)$ & \\
\hline
\end{tabular}

Table 6 shows the sub-hypothesis $\mathrm{H}_{2.2}$ testing results: Older nurses have a lower level of occupational burnout and occupational stress than younger nurses. According to age, nurses were divided into two groups: younger, up to 40 years old ( 28 nurses) and older, over 40 years old ( 32 nurses). The results show that there are no statistically significant differences in the level of occupational burnout and occupational stress between younger and older nurses, so the sub-hypothesis $\mathrm{H}_{2.2}$ has not been confirmed / is discarded.

Table 6. Results of the t-testof occupational stress and occupational burnout of nurses of different age

\begin{tabular}{|c|c|c|c|c|c|}
\hline & Age & M & SD & t- test & Sig.P \\
\hline Occupational > & $>40$ & 20,21 & 8,38 & & \\
\hline stress & $<40$ & 18,71 & 8,10 & 0,704 & 0,484 \\
\hline Occupational > & $>40$ & 69,18 & 21,01 & & \\
\hline burnout & $<40$ & 63,64 & 11,77 & 1,236 & 0,221 \\
\hline
\end{tabular}

Table 7 shows the results of the t-test of the sub-hypothesis $\mathrm{H}_{23}$ : Nurses with more years of service have a lower level of occupational burnout and occupational stress than nurses with less years of service. According to the years of service, nurses were divided into nurses with less years (under 18) of service and nurses with more years (over 18) of service. The results show that there are no statistically significant differences in the level of occupational burnout and occupational stress between nurses with more or less work experience (years of service), so the sub-hypothesis $\mathrm{H}_{2.3}$ has not been confirmed / is discarded.

Table 7. Results of the t-testof occupational stress and occupational burnout of nurses with different years of service

\begin{tabular}{llccccc}
\hline & $\begin{array}{c}\text { Years of } \\
\text { service }\end{array}$ & $\mathbf{N}$ & $\mathbf{M}$ & SD & t- test & Sig. P \\
Occupational & $>18$ & 26 & 66,88 & 20,36 & & \\
burnout & $<18$ & 34 & 66,38 & 15,09 & 0,110 & \\
Occupational & $>18$ & 26 & 19,76 & 9,12 & & 0,913 \\
stress & $<18$ & 34 & 19,32 & 8,98 & 0,206 & \\
\hline
\end{tabular}

\section{DISCUSSION}

Occupational burnout and occupational stress among health workers of different age, different years of practice, working at different departments in public health institutions, were measured in this study. The mutual relations between these two phenomena were determined with the objective of testing the previously set hypotheses. The findings of this and previous researchcoincideand show that there is a positive correlation between occupational burnout and occupational stress, which suggests that this hypothesis has been confirmed, i.e. findings confirm that increasing occupational stress increases occupational burnoutamong nursers $(3,4,5)$.

It is a fact that constant stress is possible due to poor working conditions in public health institutions, lack of resources, favorability of some employees, lack of staff and overload of nurses, etc. That leads to increased occupational burnout which reflects on their work and relation towards patients. Nurses who have high results of occupational burnout are constantly frustrated, have a problem communicating with patients and dealing with their problems, they are demotivated, cynical, unsettled. The mean findings indicate that nurses have low level of occupational stress, low depresionalization, low level of lack of personal achievement and a medium level of emotional exhaustion. Emotional exhaustion is a common occurrence among nurses due to constant exposure to stressful events, because working with patients and patience in relation to them, their problems and sufferings, rarely cause indifferent reaction. This phenomenon should be maintained at a low level, where there are no serious consequences for the psychological and physical health of employees.

Compared to sub-hypotheses, it has been shown that only the subscale of depersonalization is not related to occupational stress. Nurses who have a higher level of depersonalization have a negative, indifferent and cynical attitude towards superiors, colleagues and patients and disturb the working atmosphere. Depersonalisation is a serious problem for providing the quality health service and leaves serious consequences on the health of employees. The lack of personal achievement occurs when an employee has a distorted perception of his working ability and thinks that he/she can not perform his work obligation well, or as he/she did before. In this study, it was shown that with the increase of occupationalstress, the lack of personal achievement is being reduced, as was confirmed in other studies $(4,6)$.

Research on the correlation between occupationalstress and occupationalburnout among nurses working in private clinics was conducted by Azeem et al. on a sample of 175 respondents working in private clinics in India, where moderately low level of occupationalstress and occupationalburnout were registered. The sub-dimensionof the overload of nurses had the highest scores, and a positive correlation was found between the overload dimension and the dimension of emotional exhaustion and depersonalisation. The dimension of insufficiency is positively related to the dimensions of emotional exhaustion and depersonalization, but also negatively related to the dimension of personal realization (6).

The secondhypothesis $\mathrm{H}_{2}$ suggests that there is a difference in the level of occupationalstress and the level of occupationalburnout among nurses working in different departments, who are of different age and have different work experience, i.e. years of practice. For the first subhypothesis $\mathrm{H}_{2.1}$ claiming that nurses working on Surgery have higher results of occupationalburnout and occupationalstress than nurses working in the Gynecology and Emergency department, the results of the conducted ANOVA for 
independent groups do not show statistically significant differences in terms of occupationalburnout and occupational stress, i.e. this sub-hypothesis has not been confirmed. Previous research has shown that nurses working at the surgery department show greater results of occupationalstress and occupationalburnout than nurses working in other departments (3). The rejection of this sub-hypothesis can be owed to the fact that selected respondents from the Emergency department are always the first to respond to accidents, and gynecology in which there are many stressful interventions. The obtained findings of the t-test, compared to subhypothesis $\mathrm{H}_{2.2}$ claimingthat older nurses have lower levels of occupational burnout and occupational stress than younger nurses, do not show statistically significant differences, which means this sub-hypothesis is not confirmed. Although the connection between stress, occupational burnout and the age of nurses was found in previous relevant studies (4), this study did not confirm it. Some studies of occupationalstress among nurses employed in general hospitals in relation to demographic characteristics (age and marital status) show that younger and married nurses have a higher level of occupationalstress than older and unmarried nurses (7-12). This can be explained by the fact that older nurses used to work by outdated methods, but with the advancement of technology, development of medicine in all aspects, new methods of testing and early diagnosis of the disease, although their work is significantly facilitated, the changes make them problems. It takes more time to master new methods, more energy is involved, unlike younger nurses who do not detect the new technology change, but are regular, so they are motivated and more energetic in their work.

Results of the t-test, compared to sub-hypothesis $\mathrm{H}_{2.3}$ claiming that nurses with more years of service have a lower level of occupational burnout and occupational stress than nurses with less work experience, show no significant difference between nurses with less than 18 years of service and nurses with a higher working experience (over 18 years), i.e. this sub-hypothesis has not been confirmed. It is expected that over time, while gaining a greater work experience, nurses will courageously approach the problems they face on a daily basis, which should lead to lower levels of occupationalburnout and occupationalstress. In addition, nurses are more committed, motivated, empathic and have the desire to prove themselves when they are younger, but over the years the desire slowly decreases $(13,14)$.

\section{CONCLUSION}

The existence of a connection between occupational burnout and occupational stress among nurses working in hospital departments of surgery, gynecology and emergency has been tested. Research results show that with increased occupational stress, occupational burnoutalso increases, and that nurses have low level of occupational stress, low depresanalization level, low level of lack of personal achievement and medium level of emotional exhaustion. There were no significant differences in occupational burnout and occupational stress between nurses working in the Surgery, Gynecology and Emergency departments. No significant differences in occupational burnout and occupational stress were found in relation to the age and years of service of nurses working in the departments of Surgery, Gynecology and Emergency.

\section{LITERATURA}

1. VićentićS., JovanovićA., DunjićB., PavlovićZ., Nenadovi M, Nenadović N. Professionalstress in general practitioners and psychiatrists - the level of psycologicdistressand burnout risk, Opšta bolnica, Šabac,2009, accessed on March 25, 2018 from http://www.doiserbia.nb.rs/

2. Schaufeli W.B., Leiter M.P, Maslach C. Burnout: 35 years of research and practice.2008, accessed on March 25, 2018 from www.emeraldinsight.com/1362-0436.htm

3. WuS., Zhu W., Wang Z., Wang M., Lan Y., Relationship between burnout andoccupational stress among nurses in China, Journal of AdvachedNursing, 2007, accessed on March 16, 2018 from http: //www.ncbi.nlm.nih.gov/

4. Patrick K., Lavery J.F., Burnout in nursing, Australian Journal of AdvancedNursing, 2006, accessed on March 14, 2018 from http://www.ajan.com.au/

5. Tuna R., Baykal U., The relationship between job stress and burnout levels of oncology nurses, Nursing Department, Istanbul Medeniyet University, Istanbul, Turkey, 2014, 33-39.

6. Azeem S.M., Nazir N.A., Zaidi A., Akhtar N., Role of Stress and Burnout among Nursesin the Private Hospitals, International Journal of Academic Research inBusiness and Social Sciences, 2014, accessed on March 16, 2018 from http://hrmars.com/

7. Al-Aameri S.A., Source of job stress for nurses in public hospitals, Saudi Med J, 2003, Nov; 24(11):1183-7.

8. Dewe P.J,, O'Driscoll M.P., Cooper C.L., Theories of Psychological Stress at Work, Department of Organizational Psychology, Birkbeck, University of London, UK, 2012.

9. HandyJ.A., Theoretical and Methodological Problems Within Occupational Stress and Burnout Research, Department of Behaviour in Organizations, University of Lancaster, Bailrigg, Lancaster, U.K, 2013, accessed on March 20, 2018 from http://hum.sagepub.com/content/41/5/351.abstract

10. House J.S., Work stress and social support,Unifersity of Mitchigenm, 1944, accessed on March 16, 2018 from https: / /babel.hathitrust.org

11. Mark G.M., A.P., Smith Stress models: A review and suggested new direction, accessed on March 16, 2018 from http://psych.cf.ac.uk/ 
12. Popov S., Latovljev M.,Nedić A., Sindrom izgaranja kod zdravstvenih i prosvetnih radnika: Uloga situacionih i individualnih faktora, Psihološka istraživanja, 2015, Vol. XVIII 5-22.

13. Siegrist, J., Effort-reward imbalance at work - theory, measurement and evidence, 2012. accessed on March 14, 2018 from http://www.uniklinik-duesseldorf.de

14. ZuckM.V.,FreyJ.R., General Adaptation Syndrome, Gale Encyclopedia of Medicine, 2006, accessed on March 14, 2018 from http://www.encyclopedia.com/ 